\title{
A Review of Asenapine in the Treatment of Bipolar Disorder
}

\author{
Eduard Vieta $^{1} \cdot$ José Manuel Montes $^{2}$
}

Published online: 24 October 2017

(c) The Author(s) 2017. This article is an open access publication

\begin{abstract}
Bipolar disorder places a significant burden on the affected individuals, their family, healthcare systems and the overall economy. More treatment options are needed, especially those with better efficacy and tolerability. Asenapine is a second-generation antipsychotic approved in Europe (brand name Sycrest ${ }^{\circledR}$ ) for the treatment of moderate-to-severe manic episodes associated with bipolar I disorder in adults, and in the US (brand name Saphris ${ }^{\circledR}$ ) for the treatment of manic or mixed episodes of bipolar I disorder in adults and children aged $10-17$ years. It is the antagonistic activity at the $\mathrm{D}_{2}$ receptor that is likely responsible for the antimanic properties of asenapine. Clinical trials have demonstrated that asenapine monoand add-on therapy is effective in the short- and long-term treatment of mania associated with bipolar I disorder in adult and paediatric patients. In addition, post hoc and pooled data analyses have shown that asenapine is effective in reducing clinically significant depressive symptoms in patients with bipolar I disorder. The most common adverse events associated with asenapine are somnolence, dizziness, extrapyramidal symptoms, increased bodyweight and oral hypoesthesia. However, the incidence of these events, particularly weight gain, is generally lower than with olanzapine. In one study, asenapine has been shown to improve health-related quality of life. Economic analyses indicate that the use of asenapine can, over time, lead to a reduction in the costs of treatment.
\end{abstract}

Eduard Vieta

evieta@clinic.ub.es

1 Psychiatry and Psychology Department of the Hospital Clínic, Institute of Neuroscience, University of Barcelona, IDIBAPS, CIBERSAM, 170 Villarroel St, 08036 Barcelona, Catalonia, Spain

2 Psychiatry Section of the Ramón y Cajal University Hospital, IRYCIS, CIBERSAM, University of Alcalá, Ctra. Colmenar Viejo, km. 9,100, 28034 Madrid, Spain

\section{Key Points}

Asenapine is a second-generation atypical antipsychotic drug with demonstrated efficacy in the treatment of mania associated with bipolar I disorder.

Asenapine was also effective in the treatment of mixed states in patients with bipolar I disorder, as defined by the Diagnostic and Statistical Manual of Mental Disorders (DSM), 4th Edition, Text Revision, or major depressive disorder with mixed features, as defined by DSM-5.

Several pharmacoeconomic studies have shown that asenapine is likely to be associated with lower healthcare costs and higher quality of life.

\section{Introduction}

Bipolar disorder is a chronic affective condition characterised by episodes of mania and depression [1]. Two major types of bipolar disorder have been defined: bipolar I and bipolar II [2]. Manic episodes are the dominant feature of bipolar I; however, depressive episodes are also common. In bipolar II, manic symptoms have lower intensity and duration (hypomania), while depression is more pronounced. In addition, patients with bipolar disorder may experience episodes that combine the features of both mania and depression. The criteria for diagnosing these episodes vary. In the Diagnostic and Statistical Manual of Mental Disorders (DSM)-IV, co-occurrence of full mania and depression for more than 1 week is defined as a mixed 
state (episode), whereas in the DSM-5 it has been replaced with a 'mixed features' specifier [3].

In the general population, lifetime prevalence of bipolar spectrum disorders has been estimated at $2.4 \%$, while the prevalence of bipolar I and II is approximately 0.6 and $0.4 \%$, respectively [4]. Bipolar disorder is associated with decreased cognitive function [5] and professional ability, and increased risk of suicide and substance abuse [4]. It is characterised by a high degree of comorbidity with other psychiatric conditions [4] and places a significant burden on healthcare systems [6]. In 2010, bipolar disorder was responsible for $0.5 \%$ of the total number of disability-adjusted life-years (DALYs) worldwide, or 5.0\% of DALYs attributable to mental illness, surpassing Alzheimer's disease, Parkinson's disease and multiple sclerosis [6].

Recommended treatments for bipolar disorder include lithium, antipsychotics, anxiolytics, antidepressants, anticonvulsants, electroconvulsive therapy and their various combinations [7]. Atypical, or second-generation, antipsychotics are a class of drugs most commonly used in the treatment of bipolar disorder [8]. Asenapine is an atypical antipsychotic drug used for the treatment of mania in bipolar disorder and schizophrenia. Its active ingredient, asenapine maleate, is a synthetic tetracyclic compound that is chemically distinct from other drugs in this class. In 2009, asenapine, under the brand name Saphris ${ }^{\circledR}$, received marketing authorisation in the US for the treatment of manic or mixed episodes in adult patients with bipolar I disorder, as monotherapy or adjunctive treatment to lithium or valproate [9]. Then, in 2010, it was approved in the EU for the treatment of moderate-to-severe manic episodes in adult patients with bipolar I disorder [10]. In 2015, the US FDA also approved asenapine for the acute treatment of manic or mixed episodes of bipolar I disorder in paediatric patients (ages 10-17 years) [11]. Asenapine has been approved for the treatment of mania associated with bipolar I disorder in multiple countries in North and South America, Europe, Asia and Oceania [12]. Furthermore, asenapine is approved for the treatment of schizophrenia in the US, Canada, Australia, New Zealand and Argentina. In Europe, asenapine (brand name Sycrest ${ }^{\circledR}$ ) is available as 5 and $10 \mathrm{mg}$ rapidly dissolving tablets for sublingual administration.

The aim of this narrative review was to summarise the pharmacology, efficacy and safety of asenapine, and discuss its place in the treatment of bipolar disorder.

\section{Pharmacokinetics}

Asenapine is available in 5 and $10 \mathrm{mg}$ sublingual tablets for twice-daily administration. If administered sublingually, the bioavailability of a $5 \mathrm{mg}$ dose of asenapine is approximately 35\% [13]. Exposure increases non-linearly and approximately 1.7-fold when the dose is doubled [13]. It is recommended that eating and drinking is avoided for 10 min following administration [13]. Drinking water 2 and 5 min after administration has been shown to decrease exposure by approximately 20 and $10 \%$, respectively. These variations are within the range of intraindividual variability and may not be clinically significant [14]. Taking asenapine within $30 \mathrm{~min}$ of consuming a high-fat meal has been shown to reduce exposure by approximately $20 \%$, while consuming a high-fat meal $4 \mathrm{~h}$ after taking asenapine reduced exposure by approximately $13 \%$ [15]. Supralingual administration has been shown to be essentially bioequivalent to sublingual administration based on area under the concentration-time curve values [16]. If swallowed, the bioavailability of asenapine is approximately $2 \%$ [17]. With sublingual administration, peak plasma concentration is reached within 0.5-1.5 h [13]. Half-life is approximately $20 \mathrm{~h} \mathrm{[16],} \mathrm{and} \mathrm{steady-state}$ plasma levels are reached within 3 days of twice-daily administration [13]. Asenapine is extensively metabolised, mostly by uridine diphosphate-glucuronosyltransferase 1-4 and cytochrome P450 (CYP) 1A2 [13, 18]; however, none of the metabolites can cross the blood-brain barrier [17]. Approximately $90 \%$ of asenapine is excreted in faeces $(50 \%)$ and urine $(40 \%)$ within $96 \mathrm{~h}$ of administration $[13,18]$.

Fluvoxamine, a CYP1A2 inhibitor, has been shown to increase asenapine exposure, and coadministration should be avoided [13]. In patients with severe hepatic impairment, asenapine exposure has been shown to be approximately seven times higher than in those with normal liver function, and commensurate dose titration is advised in this population. Renal impairment does not have any substantial effect on asenapine exposure [13].

Asenapine increases blood concentrations of paroxetine [13], and should be administered with caution in combination with drugs that are both substrates and inhibitors of CYP2D6 [13].

\section{Pharmacodynamics}

The exact mechanism of action of asenapine is unknown [19]. Asenapine has been shown to interact with a wide range of receptors, including serotonin receptor subtypes $5-\mathrm{HT}_{1 \mathrm{~A}}, 5-\mathrm{HT}_{1 \mathrm{~B}}, 5-\mathrm{HT}_{2 \mathrm{~A}}, 5-\mathrm{HT}_{2 \mathrm{~B}}, 5-\mathrm{HT}_{2 \mathrm{C}}, 5-\mathrm{HT}_{5 \mathrm{~A}}, 5-\mathrm{HT}_{6}$ and 5- $\mathrm{HT}_{7}$; adrenoceptor subtypes $\alpha_{1 \mathrm{~A}}, \alpha_{2 \mathrm{~A}}, \alpha_{2 \mathrm{~B}}$ and $\alpha_{2 \mathrm{C}}$; dopamine receptor subtypes $\mathrm{D}_{1}, \mathrm{D}_{2 \mathrm{~L}}, \mathrm{D}_{2 \mathrm{~S}}, \mathrm{D}_{3}$ and $\mathrm{D}_{4}$; and histamine receptor subtypes $\mathrm{H}_{1}$ and $\mathrm{H}_{2}$. At most of these, asenapine acts as an antagonist [20], while it is likely a partial agonist at the $5-\mathrm{HT}_{1 \mathrm{~A}}$ receptor [21]. Asenapine has a low affinity for muscarinic receptors (negative logarithm of 
the binding affinity $\left.\left[\mathrm{p} K_{\mathrm{i}}\right] \leq 5\right)[20]$. Occupancy at the $\mathrm{D}_{2}$ dopamine receptor is believed to be responsible for the antimanic activity of asenapine [19]. Compared with other atypical antipsychotic drugs, the affinity of asenapine for this receptor subtype is second only to that of aripiprazole [20]. In addition, antagonist activity at 5- $\mathrm{HT}_{1 \mathrm{~A}}, 5-\mathrm{HT}_{1 \mathrm{~B}}$, $5-\mathrm{HT}_{2 \mathrm{~A}}, 5-\mathrm{HT}_{2 \mathrm{C}}, 5-\mathrm{HT}_{5 \mathrm{~A}}, 5-\mathrm{HT}_{6}$ and $5-\mathrm{HT}_{7}$ may contribute to the antimanic and antidepressant effects of asenapine [19].

\section{Therapeutic Efficacy}

\subsection{Randomised Controlled Trials and Extension Studies}

The evidence for the efficacy of asenapine in the treatment of bipolar disorder comes from a number of clinical studies that focused on patients with manic or mixed episodes (Table 1).

\subsubsection{Asenapine Versus Placebo}

An evaluation of the efficacy of flexible-dose asenapine was provided in two randomised, placebo- and olanzapinecontrolled clinical trials [22, 23]. In both studies, mean total Young Mania Rating Scale (YMRS) scores were significantly reduced at the end of the treatment period in patients who received asenapine compared with those who received placebo $[22,23]$. Significant improvements were evident early in the course of treatment (day 2) [22, 23]. The rates of response (defined as $\geq 50 \%$ reduction in total YMRS score) and remission (defined as total YMRS score $\leq 12$ after treatment) were significantly higher with asenapine compared with placebo in one of the studies [22], while they were not significantly different in the other [23]. Asenapine was associated with significant reductions in mean Clinical Global Impression-Bipolar (CGI-BP) scores compared with placebo in both studies [22, 23]. After 3 weeks of therapy, asenapine did not improve mean Montgomery-Åsberg Depression Rating Scale (MADRS) scores in either study [22, 23]. In one study, shifts in mean MADRS score from $\leq 8$ at baseline to $\geq 16$ after 3 weeks were reported in $1.6 \%$ of patients receiving asenapine, $1.1 \%$ of patients receiving olanzapine, and $2.0 \%$ of patients receiving placebo [22], while in another study, these percentages were $0,1.0$ and $4.0 \%$, respectively [23].

Asenapine was also effective at improving the symptoms of bipolar disorder when administered as a 5 or $10 \mathrm{mg}$ twice-daily fixed dose for 3 weeks [24]. Both doses produced statistically significant reductions in mean total YMRS scores at day 4 and at the end of the treatment period when compared with placebo [24]. Similarly, both doses were associated with significant reductions in mean total CGI-BP scores at day 4 and at the end of the treatment period (Table 1) [24]. Furthermore, mean MADRS scores were significantly reduced with both doses of asenapine at day 7 and at the end of the study [24].

Patients who completed the above 3-week placebocontrolled study could enter a 26-week uncontrolled extension. Those who received placebo during the 3-week trial were assigned to asenapine $5 \mathrm{mg}$ twice daily [25]. The positive effects of asenapine on efficacy parameters were maintained at the end of the extension study [25]. Mean total YMRS scores were further decreased compared with the extension study baseline in patients who received 5 and $10 \mathrm{mg}$ of asenapine, as well as in those who switched to asenapine after placebo [25]. Improvements in the rates of YMRS response and remission were observed in all treatment groups, as were improvements in the CGI-BP scores [25].

A 3-week, randomised, placebo-controlled clinical trial, plus a 40-week placebo-controlled extension study conducted in patients with bipolar I disorder with manic or mixed episodes, showed that asenapine was effective in reducing symptoms when used as add-on therapy to lithium or valproate [26]. At week 3, a significant reduction in mean total YMRS scores was observed in patients receiving asenapine compared with those receiving placebo [26]. No significant difference in mean YMRS total score was detected between patients receiving lithium and those receiving valproate [26]. Furthermore, YMRS response rate with asenapine was not significantly different to that with placebo at 3 weeks; however, at 12 weeks the response rate with asenapine was significantly higher. YMRS remission rates with asenapine were significantly higher than with placebo at weeks 3 and 12 [26]. Patients treated with asenapine had significantly lower mean total CGI-BP scores at weeks 3 and 12 compared with those who received placebo [26]. Due to the low number of patients recruited in the extension study, no robust conclusions about the efficacy of adjunctive treatment with asenapine could be drawn [26].

Asenapine has been shown to be effective in the treatment of manic or mixed episodes in paediatric patients aged 10-17 years [27, 28]. In a 3-week, randomised, placebo-controlled study, asenapine was associated with statistically significant reductions in mean total YMRS score at day 21. Reductions were observed from day 4 [27]. The rates of YMRS response were higher in patients receiving asenapine compared with placebo at day 21 [27]. At day 21 , asenapine was associated with significant reductions in mean CGI-BP scores from baseline [27]. A subsequent 50-week, placebo-controlled extension study reported that these improvements were maintained at week 26 (Table 1) [28]. 


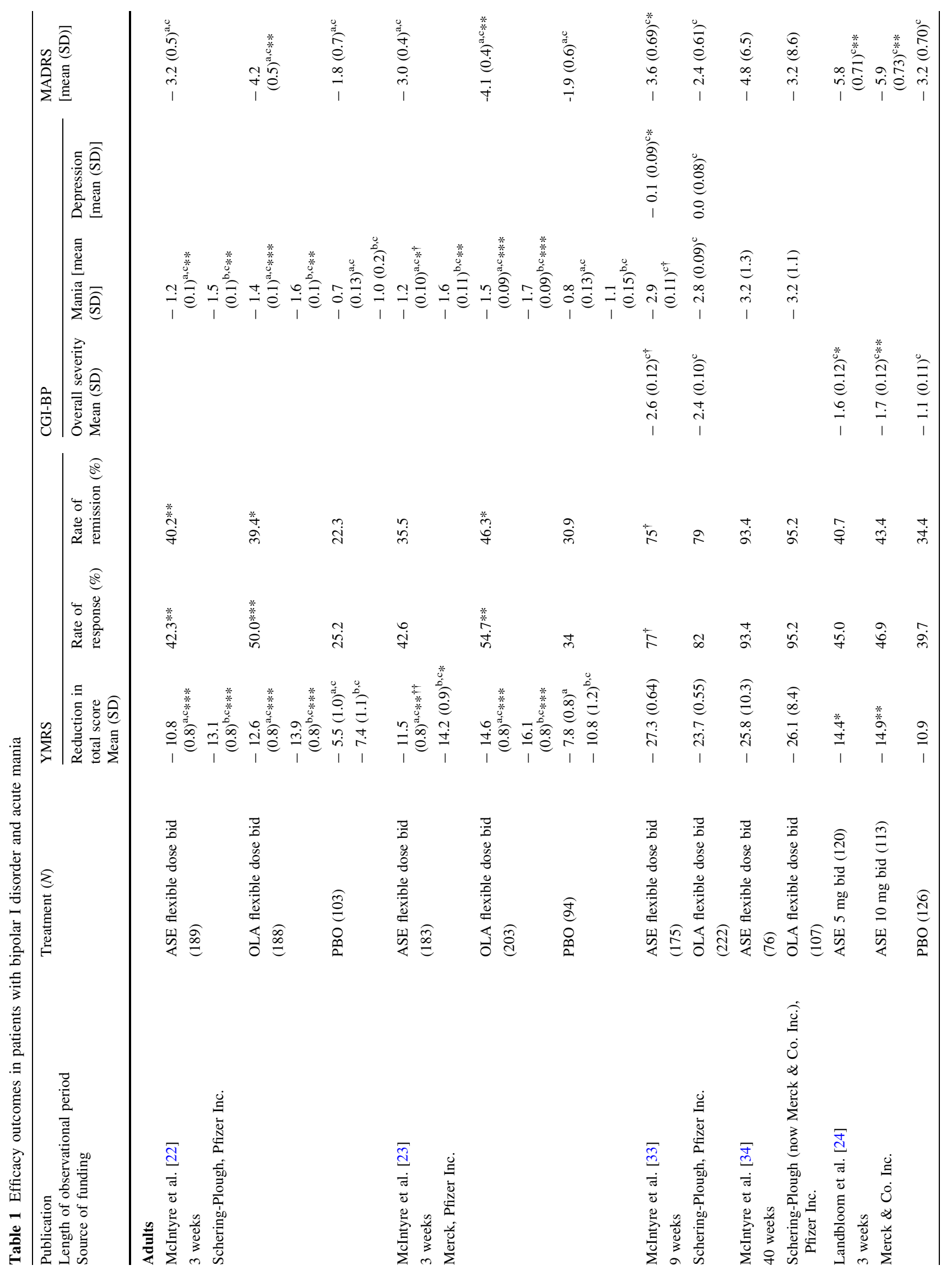




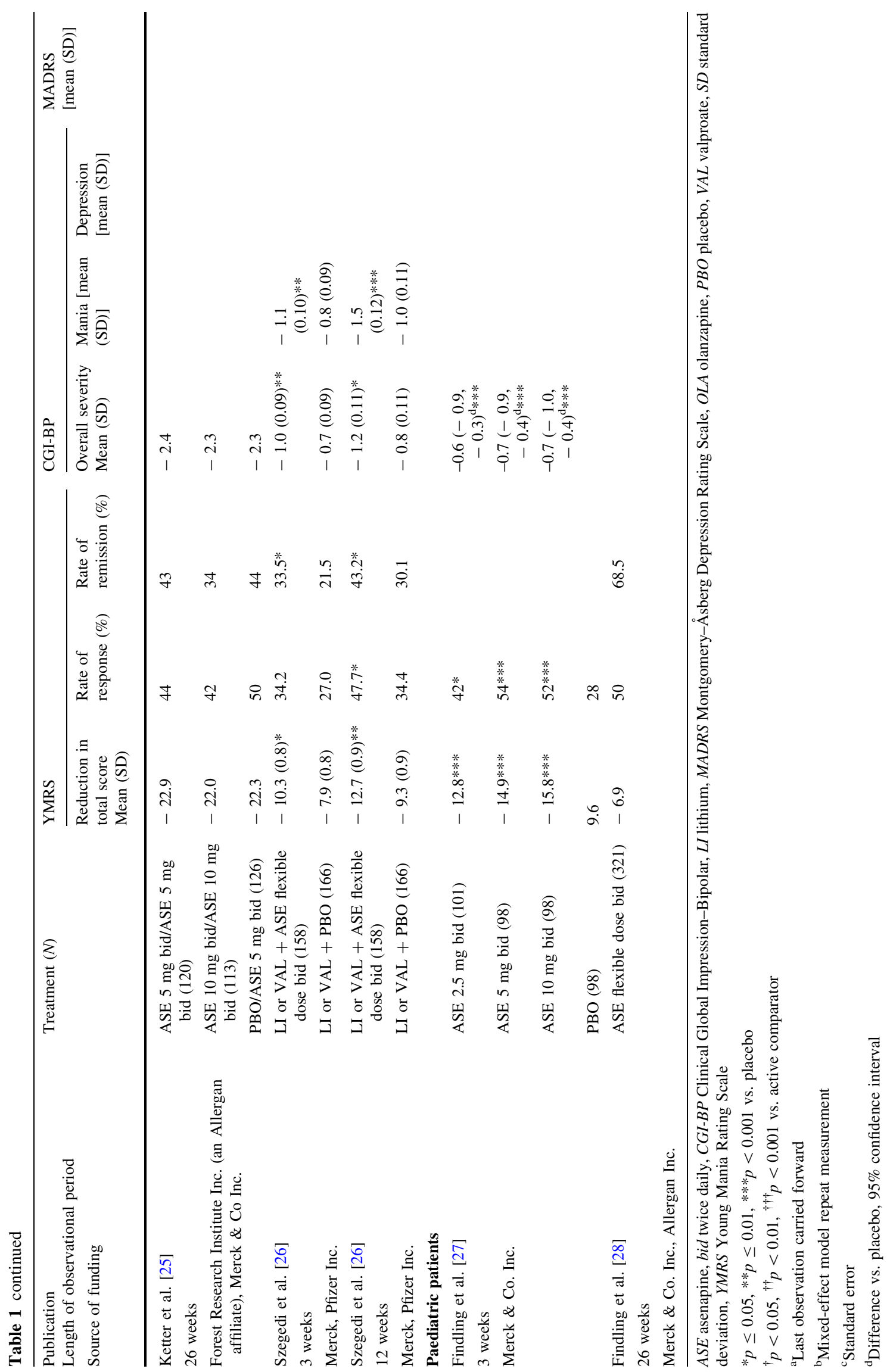


Several non-randomised studies assessed the efficacy of asenapine in the treatment of elderly patients with bipolar disorder. In a 3-week, open-label clinical study, monotherapy with asenapine was shown to be effective in the treatment of manic or mixed patients [29]. Mean total YMRS, CGI-BP mania and total MADRS scores were significantly reduced at the end of the study period [29]. A 4-week study of the effectiveness of asenapine in the acute treatment of bipolar disorder in consecutively admitted elderly patients showed significant reductions from baseline in mean total YMRS, all mean individual YMRS items and mean total CGI-BP with asenapine [30]. Additionally, another study assessed the efficacy of adjunctive treatment with asenapine in elderly patients with bipolar I and acute manic or mixed episodes [31]. At the end of the treatment period, significant reductions from baseline were observed in mean Brief Psychiatric Rating Scale [BPRS] $(p<0.05)$, total CGI $(p<0.01)$, CGI-BP mania $(p<0.05)$ and depression $(p<0.01)$ scales [31].

A naturalistic observational study assessed the efficacy of asenapine in patients with bipolar I and schizoaffective disorder [32]. After 4 weeks of treatment, significant reductions in mean Hamilton Rating Scale for Depression (HAM-D) and mean total YMRS scores were observed [32].

\subsubsection{Asenapine Versus Olanzapine}

In one of the studies by McIntyre and colleagues described above, post hoc analyses were performed to evaluate the difference in efficacy between asenapine and olanzapine. When the last observation carried forward (LOCF) method was used, reductions in YMRS scores and CGI-BP mania severity scores were greater with olanzapine than with asenapine ( $p=0.004$ and $p=0.035$, respectively). However, improvements in YMRS and CGI-BP scores were not significantly different with asenapine and olanzapine when the Mixed-Effect Model Repeated Measure model was used. Similarly, improvements in mean MADRS total score were not different in patients receiving asenapine versus olanzapine. It should be noted that the study was not powered for comparison between the active treatments [23].

Patients who completed one of the studies by McIntyre and colleagues and could benefit from continued treatment were recruited in a 9-week extension, designed to compare asenapine with olanzapine. At the end of the observational period, asenapine met the prespecified criteria for non-inferiority to olanzapine in YMRS total score, CGI-BP mania and overall illness severity scores, and YMRS response and remission rates [33]. Shifts in mean MADRS score from $\leq 8$ at baseline to $\geq 16$ at the end of the study were reported in $2.3 \%$ of patients who received asenapine and
$5.0 \%$ of those who received olanzapine [33]. Patients who completed the 9-week extension study were eligible to enter a second, 40-week extension study. At the end of the treatment period, similar reductions in YMRS scores were observed in patients treated with asenapine and those treated with olanzapine. No statistically significant differences in YMRS response and remission rates, as well as CGI-BP severity scores, were detected (Table 1) [34]. At the end of the study, no patients receiving asenapine had a shift in mean MADRS score from $\leq 8$ at baseline to $\geq 16$, while $3.0 \%$ of patients receiving olanzapine experienced such shifts [34].

\subsection{Asenapine Versus Other Antipsychotic Drugs}

The efficacy of asenapine was compared with that of several other antipsychotic drugs in a single-blind clinical study that included 155 patients with bipolar I disorder and a current manic episode [35]. Based on clinical judgement, particularly taking into account such factors as concomitant conditions and specific symptoms, 44 patients were assigned to haloperidol 3-9 mg/day, 50 patients to olanzapine $15-30 \mathrm{mg} /$ day, 16 patients to risperidone $4-9 \mathrm{mg} /$ day, 13 patients to quetiapine $600-1000 \mathrm{mg} / \mathrm{day}, 16$ patients to aripiprazole 20-30 mg/day and 16 patients to asenapine 10-20 mg/day. The outcomes were evaluated after 4 and 7 days of treatment. After 4 days of therapy, patients who received asenapine had significantly lower total YMRS scores compared with those who received haloperidol $(p=0.001)$. After 7 days of therapy, those who received asenapine had lower YMRS scores than those receiving haloperidol $(p=0.001)$, as well as those receiving olanzapine $(p=0.047)$. Also after 7 days, patients treated with asenapine achieved YMRS clinical response more often than those treated with haloperidol and risperidone $(p<0.05)$, as well as YMRS remission more often than those treated with aripiprazole, quetiapine and haloperidol $(p<0.05)$. The authors concluded that asenapine was more effective in the treatment of acute mania than other antipsychotic drugs [35].

\subsection{Post Hoc and Pooled Data Analyses}

An analysis of pooled data from the two placebo-controlled clinical trials of asenapine and olanzapine showed that both asenapine and olanzapine significantly improved each of the 11 individual items comprising the YMRS, compared with placebo at day 21. Furthermore, both treatments were associated with significant improvements in individual YMRS items, including disruptive-aggressive behaviour, content, irritability, elevated mood, sleep and speech at day 2 [36]. 
A post hoc analysis of pooled data from three randomised active-controlled trials of asenapine versus olanzapine was conducted to assess the impact of early response on treatment outcome [37]. An improvement of $\geq 15 \%$ in the total YMRS score was observed in 28 and $51 \%$ of patients who received asenapine at days 2 and 4, respectively, while a similar improvement was observed in $32 \%$ of patients who received olanzapine at day 2 and $60 \%$ of patients at day 4. Moreover, an improvement in YMRS total score at day 2 was predictive of response and remission after 3 weeks of treatment with asenapine, as well as olanzapine, and an improvement of $\geq 1$ point in CGI-BP overall illness at day 4 was predictive of response and remission at the end of week 3 , while similar improvement in the CGI-BP mania severity subscore was predictive at day 2 . The association between early response and subsequent overall response and remission was stronger with asenapine than with olanzapine [37].

The efficacy of asenapine and olanzapine in the treatment of patients with mixed episodes was analysed and compared in a post hoc analysis of two pivotal clinical trials that included 960 participants. MADRS or Positive and Negative Syndrome Scale (PANSS) items were used to detect the presence of mixed episodes. In this cohort, 34, 18 and $4.3 \%$ of patients had mild, moderate and severe depressive symptoms, respectively. MADRS remission (score $\leq 12$ ) rate with asenapine was stable at $64-67 \%$, regardless of baseline severity, while with olanzapine and placebo it decreased in patients with higher baseline MADRS score (63-38, and 49-25\%, respectively). At day 2 and across all severity groups, the reduction in YMRS score was significantly greater in patients who received asenapine compared with those who received placebo. On the other hand, the reduction in YMRS score with olanzapine was significantly greater compared with placebo only in patients with mild and moderate symptoms. With asenapine, YMRS scores continued to decrease over the entire treatment period [38].

A post hoc analysis of patients with bipolar I disorder and clinically significant depressive symptoms (which included patients who had a YMRS total score of $\geq 20$ ), who participated in placebo-controlled clinical trials of asenapine and olanzapine, demonstrated that asenapine was associated with a significant reduction in mean MADRS score compared with placebo, while the effect of olanzapine was not significantly different from that of placebo [39]. Another post hoc analysis that relied on the same data confirmed these findings. Compared with placebo, asenapine significantly improved the following depressive symptoms: sadness, reduced sleep, reduced appetite and lassitude at day 7, whereas olanzapine significantly improved lassitude when compared with placebo at day 7 . Asenapine was significantly more effective than olanzapine in improving sadness, concentration difficulties, inability to feel, and pessimistic thoughts at day 7 . At day 21, asenapine was associated with significant improvements in sadness, inner tension and inability to feel compared with placebo. Furthermore, asenapine was significantly more effective in improving inability to feel than olanzapine at day 21. Olanzapine was not associated with improvement in any of the symptoms of depression at day 21 [40].

A post hoc analysis of clinical trials of asenapine has shown that it improved health-related quality of life in patients with bipolar I disorder experiencing mixed episodes compared with placebo and olanzapine [41].

\section{Tolerability and Safety}

In patients with bipolar disorder, common adverse events (incidence of $\geq 5 \%$ and at least twice that with placebo) associated with asenapine monotherapy include somnolence (24 vs. $6 \%$; number needed to harm $[\mathrm{NNH}]=6$; 95\% confidence interval [CI] 5-9), dizziness (11 vs. 3\%; $\mathrm{NNH}=13$; 95\% CI 9-25), extrapyramidal symptoms other than akathisia (7 vs. $2 \%$; $\mathrm{NNH}=20 ; 95 \%$ CI 13-56) and increased bodyweight $(24$ vs. $<1 \%$; NNH $=6 ; 95 \%$ CI 5-9), while with asenapine add-on therapy, these common adverse events are somnolence (22\% vs. $10 \%$; $\mathrm{NNH}=9 ; 95 \%$ CI 5-25) and oral hypoesthesia (5 vs. 0\%; $\mathrm{NNH}=20 ; 95 \%$ CI 12-63) [42]. In short-term clinical studies, adverse events led to discontinuation of treatment in $10 \%$ of patients receiving asenapine monotherapy and approximately $6 \%$ of patients receiving placebo, while in the study of asenapine add-on therapy, these percentages were 16 and $11 \%$, respectively [14].

Although somnolence is the most common adverse event associated with asenapine, in short-term clinical studies in patients with bipolar disorder, the incidence of somnolence was lower with asenapine monotherapy (23.8\%) than with olanzapine monotherapy (26.4\%) [14].

The incidence of extrapyramidal adverse events in patients with bipolar disorder in short-term trials was 10.0, 4.4 and $9.4 \%$ with asenapine, placebo and olanzapine, respectively [43], and 15.7, 12.7 and $16.2 \%$, respectively, in long-term trials [43].

Asenapine is known to cause oral hypoesthesia, which has been attributed to its local anaesthetic activity [42]. Oral hypoesthesia commonly resolves within $1 \mathrm{~h}$ [13]. In clinical trials of asenapine monotherapy for acute mania in patients with bipolar disorder, the incidence of oral hypoesthesia was $4 \%$ in patients who received asenapine compared with $<1 \%$ in those who received placebo (Fig. 1) [42]. The rate of discontinuation due to oral hypoesthesia was $1.1 \%$ among patients receiving asenapine compared with $0 \%$ for placebo (Fig. 1) [42]. 


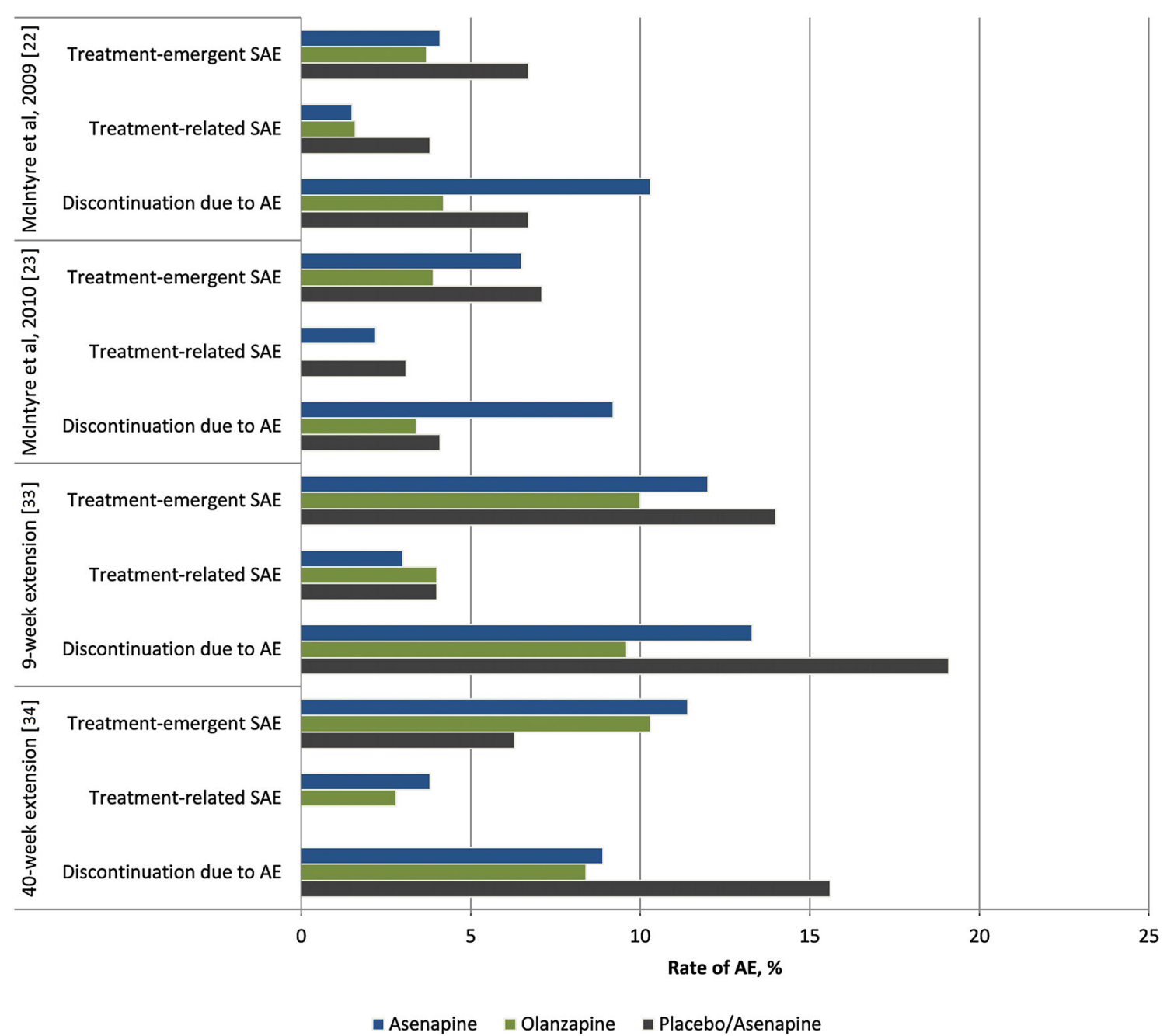

Fig. 1 Frequency of selected adverse events in clinical trials of asenapine in patients with bipolar I disorder. $A E$ adverse event, $S A E$ serious adverse event

The summary of product characteristics for asenapine [13] contains a number of warnings, one of which is for orthostatic hypotension and syncope. In short-term clinical studies that evaluated asenapine monotherapy, dizziness was reported by $11 \%$ of patients treated with asenapine compared with $3 \%$ of patients receiving placebo, and 4 and $2 \%$ of patients, respectively, in studies that evaluated addon treatment with asenapine for mania associated with bipolar I disorder. Syncope occurred in $0.03 \%$ of patients receiving asenapine monotherapy and $0 \%$ of those receiving placebo [42]. Asenapine does not appear to be associated with clinically relevant prolongation of the QT interval. Nevertheless, asenapine should be used with caution in patients with cardiovascular disease, hereditary predisposition to QT interval prolongation or those receiving medications known to cause this risk factor [13]. Another warning refers to the risk of hyperglycaemia and exacerbation of pre-existing diabetes, and monitoring is recommended in at-risk patients [13]. In short-term clinical trials, elevations of fasting serum glucose, total cholesterol and triglycerides were observed in $4.9 \%(\mathrm{NNH}=38)$, $8.7 \%(\mathrm{NHH}=1000)$ and $15.2 \%(\mathrm{NHH}=27)$ of patients who received asenapine monotherapy following treatment, respectively, compared with $2.2,8.6$ and $11.4 \%$ of patients who received placebo, respectively [42]. In short-term clinical trials, patients who received asenapine monotherapy had a mean increase in bodyweight of $1.3 \mathrm{~kg}$, while those who received placebo gained $0.2 \mathrm{~kg}$. Clinically relevant increases in bodyweight ( $\geq 7 \%$ ) occurred in $5.8 \%$ of patients on asenapine monotherapy and $0.5 \%$ of patients on placebo. In a 40-week extension study, patients continuously treated with asenapine gained a mean of $3.5 \mathrm{~kg}$, while patients treated with olanzapine gained $6.0 \mathrm{~kg}$ (Fig. 2). Clinically relevant weight gain was observed in $39.2 \%$ of patients continuously treated with asenapine compared with $55.1 \%$ of patients treated with olanzapine [42]. Hyperprolactinaemia has been reported in some patients treated with asenapine. In short-term clinical trials, 


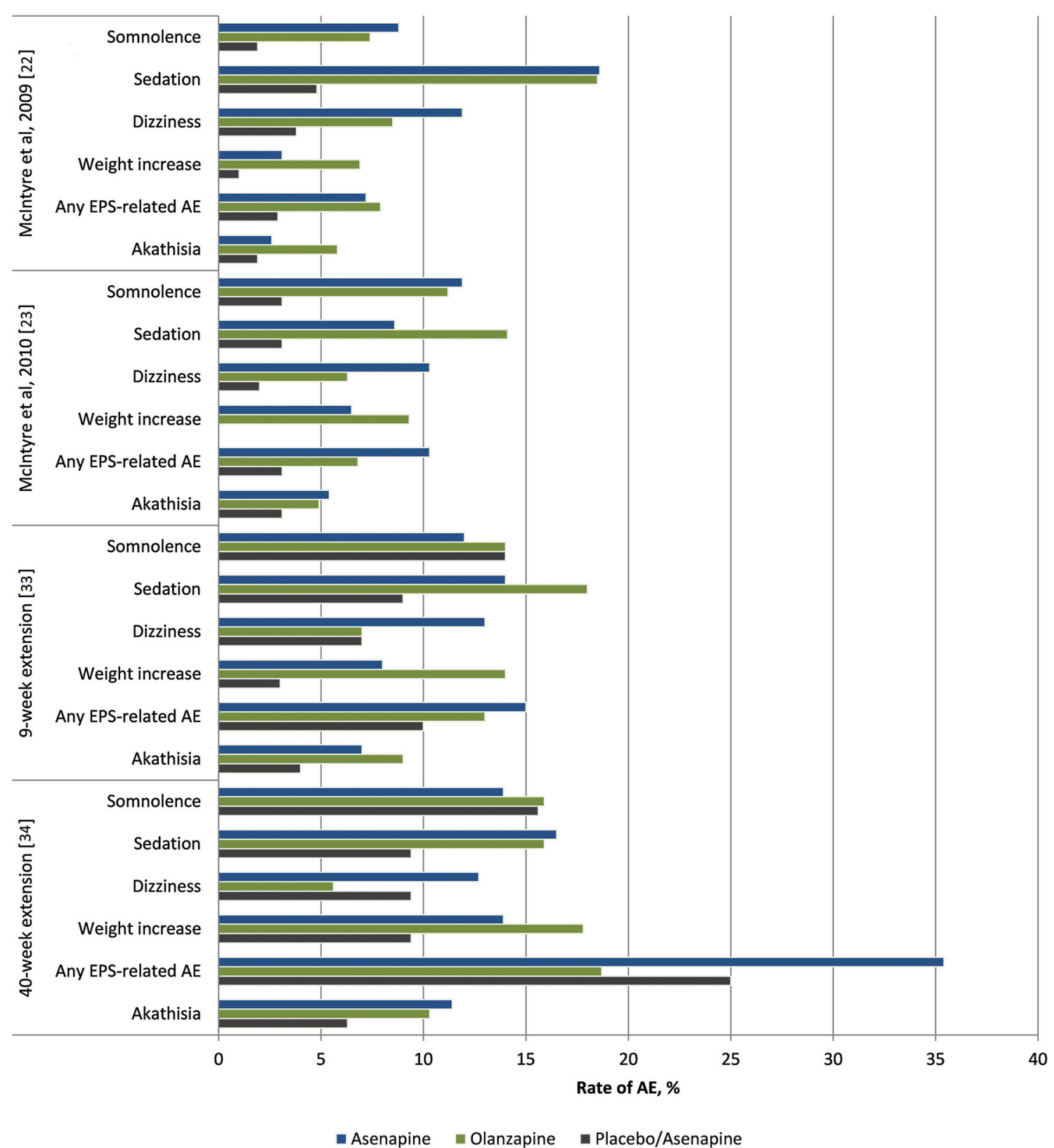

Fig. 2 Frequency of serious adverse events in clinical trials of asenapine in patients with bipolar I disorder. $A E$ adverse event, $E P S$ extrapyramidal system, $S A E$ serious adverse event

$2.3 \%(\mathrm{NHH}=63)$ of patients with bipolar disorder who received asenapine monotherapy had prolactin levels $\geq 4$ times the upper limit of the normal range compared with $0.7 \%$ of those who received placebo [42]. However, clinically significant adverse events due to hyperprolactinaemia associated with asenapine use are rare [13].

\subsection{Pooled Data Analyses}

An analysis of pooled data from two 3-week monotherapy trials and one 12-week add-on therapy trial showed that the incidence of somnolence associated with asenapine, olanzapine and placebo was 23.8, 26.4 and 6.4\%, respectively. In patients who participated in monotherapy trials, the NNH (95\% CI) for somnolence was 6 (4-9) with asenapine and 5 (4-7) with olanzapine. There was no significant difference in the time to onset of somnolence between the three treatment groups, while median duration of somnolence was 7, 8.5 and 5 days with asenapine, olanzapine and placebo, respectively. In the add-on therapy trial, the incidence of somnolence was $24.1 \%$ $(\mathrm{NNH}=7 ; 95 \%$ CI 5-18) with asenapine compared with $10.2 \%$ with placebo. While time to onset did not differ between the treatment groups, duration of somnolence 
was longer in patients treated with asenapine (12.5 vs. 7.0 days) [44].

\section{Current Use in Clinical Practice}

Asenapine is currently approved in the EU for the treatment of moderate to severe manic episodes associated with bipolar I disorder in adults [13], while in the US, it is approved for the treatment of manic or mixed episodes of bipolar I disorder in adult and paediatric patients (age 10-17 years) [9, 11]. Clinical practice guidelines from the British Association for Psychopharmacology also recommend asenapine for this indication [7], while guidelines for the treatment of major depressive episodes with mixed features by Stahl and colleagues recommend asenapine monotherapy as first-line treatment, along with lurasidone, quetiapine, quetiapine $\mathrm{XR}$, aripiprazole and ziprasidone [45]. Those cases may be particularly difficult to treat given their poor response to antidepressant drugs [46].

Available evidence, including clinical trials and post hoc and pooled data analyses, suggests that asenapine is effective in the acute and long-term treatment of manic and, in particular, mixed episodes in patients with bipolar disorder. The goal of the MANACOR study (MANía Aguda y COnsumo de Recursos, acute mania and health resource consumption), which combined prospective and retrospective data collection and analysis, was to estimate the healthcare resource consumption and direct costs associated with manic episodes in real-world clinical practice in Spain [47]. An analysis of data revealed the prescribing patterns associated with asenapine [48]. Patients who were prescribed adjunctive asenapine tended to have less severe manic episodes, fewer psychotic manifestations and a more complex clinical history, in particular, of mixed episodes and sexual dysfunction, compared with those receiving other adjunctive antipsychotics [48]. The DSM-5 defines mixed states more broadly than DSMIV, allowing for mania with some depressive symptoms, depression with some manic symptoms, and hypomania with some depressive symptoms, which are diagnosed using a dimensional approach [49, 50]. The IMPACT (Investigating Manic Phases And Current Trends) of Bipolar international multicentre study utilised a 54-item online questionnaire, which was administered to 700 patients with bipolar I disorder, to assess the specific phenomenology of depressive symptoms in patients who had a manic episode with depressive features, as defined by the DSM-5 [3]. The results indicate that patients who reported to have at least three depressive symptoms during a manic episode also commonly experienced anxiety with irritability or agitation [3]. Based on these findings, as well as those from post hoc analyses of the pivotal clinical trials of asenapine [51], some authors suggest that a combination of anxiety, irritability and agitation symptoms represents the most sensitive approach to detecting mixed states [52].

Although oral hypoesthesia was relatively uncommon in randomised controlled trials of asenapine, when healthy male participants were asked about oral hypoesthesia in a pharmacokinetics study, $22.2 \%$ reported experiencing this adverse event [16]. These data suggest that in real-world practice, the prevalence of oral hypoesthesia may in fact be much higher than in randomised clinical trials. It is recommended that patients are informed about the possibility of this adverse event before taking asenapine to avoid any negative effect on adherence [42].

The available data on the safety of asenapine suggest that it is less likely to weight gain than olanzapine. This is particularly important because weight gain is one of the adverse events most likely to result in treatment noncompliance [53]. Furthermore, asenapine has been shown to significantly improve health-related quality of life compared with olanzapine [41]. Pharmacoeconomic analyses support this conclusion and suggest that the use of asenapine is likely to reduce healthcare-related costs in patients with bipolar disorder.

\section{Discussion}

Asenapine is a second-generation atypical antipsychotic drug that is approved for the treatment of manic episodes in patients with bipolar I disorder. It is available in 5 and $10 \mathrm{mg}$ tablets for twice-daily sublingual administration. The results of a study that compared $5 \mathrm{mg}$ asenapine twice daily and $10 \mathrm{mg}$ asenapine once daily at bedtime suggest that the latter regimen, by reducing the rate of daytime sedation, may improve adherence [54]. Patients are recommended to avoid eating and drinking for $10 \mathrm{~min}$ after taking asenapine, and eating a meal within $4 \mathrm{~h}$ has been shown to reduce exposure. Therefore, adherence to asenapine may be negatively affected by the limitations on when and how often it must be taken.

The efficacy of asenapine in the treatment of manic episodes in patients with bipolar I disorder has been the subject of several studies. Asenapine was consistently associated with improvements in acute mania, as measured by YMRS and CGI-BP in paediatric (age 10-17 years), adult and elderly patients. A number of long-term extension studies have also shown that the effects of asenapine are maintained for up to 40 weeks. The efficacy of asenapine was either similar or superior to that of olanzapine. Of note, asenapine did not produce significant improvements in MADRS scores in randomised controlled trials; however, in a post hoc analysis, asenapine was shown to be effective in reducing the symptoms of depression in 
patients with bipolar I disorder and a current manic episode. In order to properly assess the place of asenapine in the antipsychotic armamentarium, additional direct comparisons with other antipsychotic drugs, in the acute as well as long-term treatment of mania, are required.

The use of asenapine is associated with a number of adverse events, including somnolence, dizziness, extrapyramidal symptoms and increased bodyweight. In a meta-analysis of clinical trials of various antipsychotics, the authors concluded that asenapine presents a mid-level risk of weight gain compared with other drugs in this class. Clozapine and olanzapine were found to have the highest risk of weight gain, while aripiprazole, lurasidone and ziprasidone are associated with a lower risk of weight gain than asenapine [55]. The Summary of Product Characteristics for asenapine contains several warnings, including a warning for orthostatic hypotension and syncope, and for hyperglycaemia and exacerbation of pre-existing diabetes. The risk of blood lipid and glucose abnormalities associated with asenapine is relatively low and is less serious than with clozapine and olanzapine [56]. Another adverse event observed with asenapine is oral hypoesthesia. Although the frequency of this event was relatively low in randomised controlled trials, it is likely higher in realworld practice. The safety and tolerability profile of asenapine appears to be similar to that of olanzapine. As with efficacy, additional studies that directly compare the safety of asenapine with other antipsychotic drugs may allow for a more comprehensive assessment.

An economic evaluation of the use of asenapine in the treatment of patients with mixed episodes associated with bipolar I disorder, based on data from the Ares trial programme and extrapolated using a Markov model, was conducted [57]. Over the course of 5 years, asenapine generated 0.0187 more quality-adjusted life-years (QALYs) compared with olanzapine for every additional £24. This was primarily driven by an earlier response to asenapine [57]. This model was adapted for use in another study that aimed to estimate the cost effectiveness of asenapine compared with olanzapine in the treatment of patients with bipolar I disorder and a current manic episode in the context of Italian National Health Service [58]. Asenapine was found to be associated with lower direct costs, which was mostly due to the savings from hospitalisations avoided. Asenapine was also associated with higher quality of life compared with olanzapine [58]. The cost effectiveness of asenapine therapy for mania in patients with bipolar I disorder was also evaluated in the context of Canadian practice, using a decision-tree account of the probability of extrapyramidal symptoms, switching to a different antipsychotic drug and gaining weight, and the Markov model to account for long-term metabolic complications such as diabetes, hypertension, coronary heart disease and stroke. Asenapine was associated with lower costs and generated more QALYs [59]. An analysis of a large US medical insurance database that included claims 6 months before and after the introduction of asenapine was carried out to assess the healthcare utilisation costs [60]. While pharmacy-related costs increased, these costs were offset by the reduced costs in admissions and emergency department visits following the introduction of asenapine [60]. Asenapine also reduced costs associated with the management of manic episodes in the MANACOR study [47].

\section{Conclusions}

Asenapine is effective in the acute and long-term treatment of manic and mixed episodes associated with bipolar disorder. It produces a rapid reduction in the symptoms of mania and is particularly effective in the treatment of mixed episodes. Furthermore, asenapine is effective in alleviating the symptoms of depression in patients with bipolar I disorder during mania. The risk of weight gain is lower with asenapine than with olanzapine. In addition, asenapine has been shown to improve health-related quality of life and reduce hospitalisation costs.

Acknowledgements The authors would like to thank Georgii Filatov of Springer Healthcare Communications, who wrote the outline and subsequent drafts of this article. Medical writing assistance was funded by Lundbeck.

\section{Compliance with Ethical Standards}

Funding The preparation of this article was funded by Lundbeck.

Conflict of interest Dr. Vieta has received grants from and served as a consultant, advisor or CME speaker for the following entities: ABBiotics, Aequus, Adamed, Alexza, Allergan, Almirall, AstraZeneca, Bial, Bristol-Myers Squibb, Dainippon Sumitomo Pharma, Elan, Eli Lilly, Ferrer, Forest Research Institute, Gedeon Richter, Glaxo-SmithKline, Janssen-Cilag, Jazz, Johnson \& Johnson, Lundbeck, Merck, Novartis, Organon, Otsuka, Pfizer, Pierre-Fabre, Qualigen, Roche, Sanofi-Aventis, Servier, Shering-Plough, Shire, Solvay, Sunovion, Takeda, Telefónica, Teva, the Spanish Ministry of Science and Innovation (CIBERSAM), the Seventh European Framework Programme (ENBREC), the Stanley Medical Research Institute, United Biosource Corporation, and Wyeth. Dr. Montes has received grants from and served as consultant, advisor or CME speaker for the following entities: Almirall, AstraZeneca, Bristol-Myers Squibb, Eli Lilly, Ferrer, Glaxo-Smith-Kline, Janssen-Cilag, Lundbeck, Otsuka, Pfizer, Qualigen, Sanofi-Aventis, Servier and the Spanish Ministry of Science and Innovation (CIBERSAM).

Open Access This article is distributed under the terms of the Creative Commons Attribution-NonCommercial 4.0 International License (http://creativecommons.org/licenses/by-nc/4.0/), which permits any noncommercial use, distribution, and reproduction in any medium, provided you give appropriate credit to the original 
author(s) and the source, provide a link to the Creative Commons license, and indicate if changes were made.

\section{References}

1. Grande I, Berk M, Birmaher B, Vieta E. Bipolar disorder. Lancet. 2016;387:1561-72.

2. American Psychiatric Association. Bipolar and Related Disorders. In: American Psychiatric Association (eds). Diagnostic and Statistical Manual of Mental Disorders. 5th ed. Washington, DC. American Psychiatric Assocation; 2013. Available at: http://dsm. psychiatryonline.org/doi/full/10.1176/appi.books.9780890425596. dsm03. Accessed 11 Jan 2017.

3. Vieta E, Grunze H, Azorin JM, Fagiolini A. Phenomenology of manic episodes according to the presence or absence of depressive features as defined in DSM-5: Results from the IMPACT self-reported online survey. J Affect Disord. 2014;156:206-13.

4. Merikangas KR, Jin R, He JP, Kessler RC, Lee S, Sampson NA, et al. Prevalence and correlates of bipolar spectrum disorder in the world mental health survey initiative. Arch Gen Psychiatry. 2011;68:241-51.

5. Bortolato B, Miskowiak KW, Kohler CA, Vieta E, Carvalho AF. Cognitive dysfunction in bipolar disorder and schizophrenia: a systematic review of meta-analyses. Neuropsychiatr Dis Treat. 2015;11:3111-25.

6. Whiteford HA, Ferrari AJ, Degenhardt L, Feigin V, Vos T. The global burden of mental, neurological and substance use disorders: an analysis from the Global Burden of Disease Study 2010. PLoS One. 2015;10:e0116820.

7. Goodwin GM, Haddad PM, Ferrier IN, Aronson JK, Barnes T, Cipriani A, et al. Evidence-based guidelines for treating bipolar disorder: revised third edition recommendations from the British Association for Psychopharmacology. J Psychopharmacol. 2016;30:495-553.

8. Vieta E, Langosch JM, Figueira ML, Souery D, Blasco-Colmenares E, Medina E, et al. Clinical management and burden of bipolar disorder: results from a multinational longitudinal study (WAVE-bd). Int J Neuropsychopharmacol. 2013;16:1719-32.

9. Food and Drug Administration. Approval letter. Rockville, MD. Food and Drug Administration; 2009. Available at: https://www. accessdata.fda.gov/drugsatfda_docs/nda/2009/022117s000_Approv. pdf. Accessed 14 Sep 2017.

10. European Medicines Agency. Sycrest: EPAR - summary for the public. London: European Medicines Agency; 2015.

11. Allergan. SAPHRIS ${ }^{\circledR}$ (asenapine) $2.5 \mathrm{mg}$ sublingual tablets for the acute treatment of manic or mixed episodes of bipolar i disorder in pediatric patients (ages 10-17). Available at: https:// www.allergan.com/news/news/thomson-reuters/actavis-receivesfda-approval-of-saphris-for-pedia. Accessed 14 Sept 2017.

12. AdisInsight Drugs. Asenapine. 2017. Available at: http:// adisinsight.springer.com/drugs/800001124. Accessed 14 Sept 2017.

13. European Medicines Agency. Sycrest: EPAR-product information. London: European Medicines Agency; 2010.

14. Citrome L. Asenapine review, part I: chemistry, receptor affinity profile, pharmacokinetics and metabolism. Expert Opin Drug Metab Toxicol. 2014;10:893-903.

15. Dogterom P, de Greef R, Peeters PA. The effect of food on the high clearance drug asenapine after sublingual administration to healthy male volunteers. Eur J Clin Pharmacol. 2015;71:65-74.

16. Gerrits M, de Greef R, Peeters P. Effect of absorption site on the pharmacokinetics of sublingual asenapine in healthy male subjects. Biopharm Drug Dispos. 2010;31:351-7.
17. Citrome L. Asenapine for schizophrenia and bipolar disorder: a review of the efficacy and safety profile for this newly approved sublingually absorbed second-generation antipsychotic. Int J Clin Pract. 2009;63:1762-84.

18. van de Wetering-Krebbers SF, Jacobs PL, Kemperman GJ, Spaans E, Peeters PA, Delbressine LP, et al. Metabolism and excretion of asenapine in healthy male subjects. Drug Metab Dispos. 2011;39:580-90.

19. Reynolds GP. Receptor mechanisms of antipsychotic drug action in bipolar disorder-focus on asenapine. Ther Adv Psychopharmacol. 2011;1:197-204.

20. Shahid M, Walker GB, Zorn SH, Wong EH. Asenapine: a novel psychopharmacologic agent with a unique human receptor signature. J Psychopharmacol. 2009;23:65-73.

21. Ghanbari R, El Mansari M, Shahid M, Blier P. Electrophysiological characterization of the effects of asenapine at 5-HT(1A), 5-HT(2A), alpha(2)-adrenergic and $\mathrm{D}(2)$ receptors in the rat brain. Eur Neuropsychopharmacol. 2009;19:177-87.

22. McIntyre RS, Cohen M, Zhao J, Alphs L, Macek TA, Panagides J. A 3-week, randomized, placebo-controlled trial of asenapine in the treatment of acute mania in bipolar mania and mixed states. Bipolar Disord. 2009;11:673-86.

23. McIntyre RS, Cohen M, Zhao J, Alphs L, Macek TA, Panagides J. Asenapine in the treatment of acute mania in bipolar I disorder: a randomized, double-blind, placebo-controlled trial. J Affect Disord. 2010;122:27-38.

24. Landbloom RL, Mackle M, Wu X, Kelly L, Snow-Adami L, McIntyre RS, et al. Asenapine: efficacy and safety of 5 and $10 \mathrm{mg}$ bid in a 3-week, randomized, double-blind, placebo-controlled trial in adults with a manic or mixed episode associated with bipolar I disorder. J Affect Disord. 2016;190:103-10.

25. Ketter TA, Durgam S, Landbloom R, Mackle M, Wu X, Mathews M. Long-term safety and tolerability of asenapine: a doubleblind, uncontrolled, long-term extension trial in adults with an acute manic or mixed episode associated with bipolar I disorder. J Affect Disord. 2017;207:384-92.

26. Szegedi A, Calabrese JR, Stet L, Mackle M, Zhao J, Panagides J. Asenapine as adjunctive treatment for acute mania associated with bipolar disorder: results of a 12-week core study and 40-week extension. J Clin Psychopharmacol. 2012;32:46-55.

27. Findling RL, Landbloom RL, Szegedi A, Koppenhaver J, Braat S, Zhu Q, et al. Asenapine for the acute treatment of pediatric manic or mixed episode of bipolar I disorder. J Am Acad Child Adolesc Psychiatry. 2015;54:1032-41.

28. Findling RL, Landbloom RL, Mackle M, Wu X, Snow-Adami L, Chang $\mathrm{K}$, et al. Long-term safety of asenapine in pediatric patients diagnosed with bipolar i disorder: a 50-week open-label, flexible-dose trial. Paediatr Drugs. 2016;18:367-78.

29. Barak Y, Finkelstein I, Pridan S. The geriatric mania asenapine study (GeMS). Arch Gerontol Geriatr. 2016;64:111-4.

30. Baruch Y, Tadger S, Plopski I, Barak Y. Asenapine for elderly bipolar manic patients. J Affect Disord. 2013;145:130-2.

31. Sajatovic M, Dines P, Fuentes-Casiano E, Athey M, Cassidy KA, Sams J, et al. Asenapine in the treatment of older adults with bipolar disorder. Int J Geriatr Psychiatry. 2015;30:710-9.

32. Gramaglia C, Rizza MC, Gattoni E, Gambaro E, Di Marco S, Coppola I, et al. Asenapine in clinical practice: preliminary results from a naturalistic observational study. Riv Psichiatr. 2014;49:241-6.

33. McIntyre RS, Cohen M, Zhao J, Alphs L, Macek TA, Panagides J. Asenapine versus olanzapine in acute mania: a double-blind extension study. Bipolar Disord. 2009;11:815-26.

34. McIntyre RS, Cohen M, Zhao J, Alphs L, Macek TA, Panagides J. Asenapine for long-term treatment of bipolar disorder: a double-blind 40-week extension study. J Affect Disord. 2010;126:358-65. 
35. Buoli M, Esposito CM, Godio M, Caldiroli A, Serati M, Altamura AC. Have antipsychotics a different speed of action in the acute treatment of mania? A single-blind comparative study. J Psychopharmacol. 2017:269881117705098.

36. Cazorla P, Zhao J, Mackle M, Szegedi A. Asenapine effects on individual Young Mania Rating Scale items in bipolar disorder patients with acute manic or mixed episodes: a pooled analysis. Neuropsychiatr Dis Treat. 2013;9:409-13.

37. Szegedi A, Zhao J, McIntyre RS. Early improvement as a predictor of acute treatment outcome in manic or mixed episodes in bipolar-1 disorder: a pooled, post hoc analysis from the asenapine development program. J Affect Disord. 2013;150:745-52.

38. McIntyre RS, Tohen M, Berk M, Zhao J, Weiller E. DSM-5 mixed specifier for manic episodes: evaluating the effect of depressive features on severity and treatment outcome using asenapine clinical trial data. J Affect Disord. 2013;150:378-83.

39. Szegedi A, Zhao J, van Willigenburg A, Nations KR, Mackle M, Panagides J. Effects of asenapine on depressive symptoms in patients with bipolar I disorder experiencing acute manic or mixed episodes: a post hoc analysis of two 3 -week clinical trials. BMC Psychiatry. 2011;11:101.

40. Berk M, Tiller JW, Zhao J, Yatham LN, Malhi GS, Weiller E. Effects of asenapine in bipolar I patients meeting proxy criteria for moderate-to-severe mixed major depressive episodes: a post hoc analysis. J Clin Psychiatry. 2015;76:728-34.

41. Michalak EE, Guiraud-Diawara A, Sapin C. Asenapine treatment and health-related quality of life in patients experiencing bipolar I disorder with mixed episodes: post-hoc analyses of pivotal trials. Curr Med Res Opin. 2014;30:711-8.

42. Citrome L. Asenapine review, part II: clinical efficacy, safety and tolerability. Expert Opin Drug Saf. 2014;13:803-30.

43. European Medicines Agency. Sycrest: assessment report. London: European Medicines Agency; 2010.

44. Gao K, Mackle M, Cazorla P, Zhao J, Szegedi A. Comparison of somnolence associated with asenapine, olanzapine, risperidone, and haloperidol relative to placebo in patients with schizophrenia or bipolar disorder. Neuropsychiatr Dis Treat. 2013;9:1145-57.

45. Stahl SM, Morrissette DA, Faedda G, Fava M, Goldberg JF, Keck $\mathrm{PE}$, et al. Guidelines for the recognition and management of mixed depression. CNS Spectr. 2017;22:203-19.

46. Barbuti M, Pacchiarotti I, Vieta E, Azorin JM, Angst J, Bowden $\mathrm{CL}$, et al. Antidepressant-induced hypomania/mania in patients with major depression: evidence from the BRIDGE-II-MIX study. J Affect Disord. 2017;219:187-92.

47. Hidalgo-Mazzei D, Undurraga J, Reinares M, Bonnín Cdel M, Sáez C, Mur M, et al. The real world cost and health resource utilization associated to manic episodes: the MANACOR study. Rev Psiquiatr Salud Ment. 2015;8:55-64.

48. Grande I, Hidalgo-Mazzei D, Nieto E, Mur M, Saez C, Forcada I, et al. Asenapine prescribing patterns in the treatment of manic inand outpatients: results from the MANACOR study. Eur Psychiatry. 2015;30:528-34.

49. Vieta E, Valentí M. Mixed states in DSM-5: implications for clinical care, education, and research. J Affect Disord. 2013;148:28-36.

50. Solé E, Garriga M, Valentí M, Vieta E. Mixed features in bipolar disorder. CNS Spectr. 2017;22:134-40.

51. Azorin JM, Sapin C, Weiller E. Effect of asenapine on manic and depressive symptoms in bipolar I patients with mixed episodes: results from post hoc analyses. J Affect Disord. 2013;145:62-9.

52. Young AH, Eberhard J. Evaluating depressive symptoms in mania: a naturalistic study of patients with bipolar disorder. Neuropsychiatr Dis Treat. 2015;11:1137-43.

53. Levin JB, Krivenko A, Howland M, Schlachet R, Sajatovic M. Medication adherence in patients with bipolar disorder: a comprehensive review. CNS Drugs. 2016;30:819-35.

54. Sun X, Hamer R, McEvoy J. Asenapine once daily versus twice daily: impact on patient acceptance in a randomized, open-label, 14-day clinical trial. J Clin Psychiatry. 2015;76:992-3.

55. Musil R, Obermeier M, Russ P, Hamerle M. Weight gain and antipsychotics: a drug safety review. Expert Opin Drug Saf. 2015;14:73-96.

56. De Hert M, Yu W, Detraux J, Sweers K, van Winkel R, Correll CU. Body weight and metabolic adverse effects of asenapine, iloperidone, lurasidone and paliperidone in the treatment of schizophrenia and bipolar disorder: a syLstematic review and exploratory meta-analysis. CNS Drugs. 2012;26:733-59.

57. Sawyer L, Azorin JM, Chang S, Rinciog C, Guiraud-Diawara A, Marre C, et al. Cost-effectiveness of asenapine in the treatment of bipolar I disorder patients with mixed episodes. J Med Econ. 2014;17:508-19.

58. Caresano C, Di Sciascio G, Fagiolini A, Maina G, Perugi G, Ripellino C, et al. Cost-effectiveness of asenapine in the treatment of patients with bipolar I disorder with mixed episodes in an Italian context. Adv Ther. 2014;31:873-90.

59. Lachaine J, Beauchemin C, Mathurin K, Gilbert D, Beillat M. Cost-effectiveness of asenapine in the treatment of bipolar disorder in Canada. BMC Psychiatry. 2014;14:16.

60. Chitnis A, Wang R, Sun SX, Dixit S, Tawah A, Boulanger L. Impact of initiation of asenapine on patterns of utilization and cost of healthcare resources associated with the treatment of bipolar I disorder. J Med Econ. 2015;18:863-70. 